\title{
RADIO FREQUENCY MODELS OF NOVAE IN ERUPTION. I. THE FREE-FREE PROCESS IN BIPOLAR MORPHOLOGIES
}

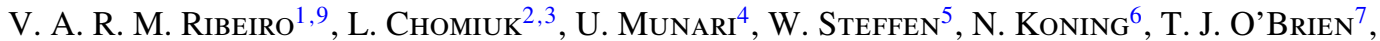 \\ T. SimON ${ }^{1}$, P. A. WOUdT ${ }^{1}$, AND M. F. BodE ${ }^{8}$ \\ ${ }^{1}$ Astrophysics, Cosmology and Gravity Centre, Department of Astronomy, University of Cape Town, \\ Private Bag X3, Rondebosch 7701, South Africa; vribeiro@ast.uct.ac.za \\ 2 Department of Physics and Astronomy, Michigan State University, East Lansing, MI 48824, USA \\ ${ }^{3}$ National Radio Astronomy Observatory, 520 Edgemont Rd, Charlottesville, VA, USA \\ ${ }^{4}$ NAF Astronomical Observatory of Padova, I-36012 Asiago (VI), Italy \\ ${ }^{5}$ Instituto de Astronomía, Universidad Nacional Autónoma de México, C.P. 22860, Ensenada, Mexico \\ ${ }^{6}$ Department of Physics \& Astronomy, University of Calgary, Calgary, Alberta T2N 1N4, Canada \\ ${ }^{7}$ Jodrell Bank Centre for Astrophysics, University of Manchester, Manchester M13 9PL, UK \\ ${ }^{8}$ Astrophysics Research Institute, Liverpool John Moores University, IC2 Liverpool Science Park, 146 Brownlow Hill, L3 5RF, UK \\ Received 2014 March 10; accepted 2014 July 8; published 2014 August 14
}

\begin{abstract}
Observations of novae at radio frequencies provide us with a measure of the total ejected mass, density profile, and kinetic energy of a nova eruption. The radio emission is typically well characterized by the free-free emission process. Most models to date have assumed spherical symmetry for the eruption, although for as long as there have been radio observations of these systems, it has been known that spherical eruptions are too simplistic a geometry. In this paper, we build bipolar models of the nova eruption, assuming the free-free process, and show the effects of varying different parameters on the radio light curves. The parameters considered include the ratio of the minorto major-axis, the inclination angle, and shell thickness. We also show the uncertainty introduced when fitting spherical-model synthetic light curves to bipolar-model synthetic light curves. We find that the optically thick phase rises with the same power law $\left(S_{v} \propto t^{2}\right)$ for both the spherical and bipolar models. In the bipolar case, there is a "plateau" phase-depending on the thickness of the shell as well as the ratio of the minor- to major-axis-before the final decline, which follows the same power law $\left(S_{v} \propto t^{-3}\right)$ as in the spherical case. Finally, fitting spherical models to the bipolar-model synthetic light curves requires, in the worst-case scenario, doubling the ejected mass, more than halving the electron temperature, and reducing the shell thickness by nearly a factor of 10 . This implies that in some systems we have been over-predicting the ejected masses and under-predicting the electron temperature of the ejecta.
\end{abstract}

Key words: novae, cataclysmic variables - radio continuum: stars

Online-only material: color figures, animation

\section{INTRODUCTION}

A nova eruption occurs in a binary system following extensive accretion of hydrogen-rich material onto the surface of a white dwarf primary from a less-evolved secondary star. The eruption is well established to be a thermonuclear runaway on the surface of the white dwarf (see, e.g., Starrfield et al. 2008). The eruption ejects of the order of $10^{-7}$ to $10^{-3} M_{\odot}$ of matter at velocities of the order of hundreds to thousands of kilometers per second (e.g., Bode \& Evans 2008; Bode 2010). Since the white dwarf is not destroyed in the explosion, it may accrete more matter from the secondary star, either a main-sequence, sub-giant, or giant star, and go into a cycle of eruptions. These eruptions can recur on timescales of years to millions of years, governed by properties of the white dwarf and the accretion (Starrfield et al. 1985; Truran \& Livio 1986; Yaron et al. 2005). Therefore, depending on the details of the white dwarf, including its composition and mass, plus the accretion rate and ejected mass, we may expect the white dwarf either to grow in mass or not. If the mass of the white dwarf does grow (e.g., Newsham et al. 2013), then it may reach the Chandrasekhar limit and end in an accretion-induced collapse to form a neutron star (in the case of an ONe white dwarf; see, e.g., Ritossa et al. 1996), or grow

\footnotetext{
9 South African Square Kilometer Array Fellow.
}

and explode as a Type Ia supernova (in the case of a $\mathrm{CO}$ white dwarf; for an extensive review see Di Stefano et al. 2013).

Novae are now known to emit at all wavelengths, from $\gamma$-rays to radio, with each providing vital information about the system parameters at the onset of the eruption. For example, observations at radio frequencies are of particular interest due to the fact that we can derive global properties of the ejecta, since radio emission is dominated by simple thermal free-free emission and does not suffer from interstellar extinction. Radio light curves, therefore, provide us with a measure of the total ejected mass, density profiles, and kinetic energy (Seaquist \& Bode 2008; Hjellming 1996), and the distance once the ejection velocity is known.

The first radio light curves of novae (HR Del, FH Ser, and V1500 Cyg; Hjellming \& Wade 1970; Wade \& Hjellming 1971) were interpreted in terms of spherically symmetric ejecta with emission arising from the free-free process. The observed radio light curves, with spherical ejecta or otherwise, were described as arising from either a finite shell with a homologous expansion (Seaquist \& Palimaka 1977; Hjellming et al. 1979), or a wind with a constant velocity and mass-loss rate (Kwok 1983). The typical model consists of a $1 / r^{2}$ density profile with a constant temperature with time and radius in the ejecta, where $T \sim 10^{4} \mathrm{~K}$.

At the beginning of the eruption, the spherically symmetric shell is optically thick at all frequencies, increasing in flux 
density (proportional to the surface area of the shell, as seen on the plane of the sky) and following the Planck function, hence with a spectral index of $\alpha=2.0$ (where $S_{v} \propto v^{\alpha}$ is the flux density and $v$ is the observed frequency). The flux density at this stage depends on the distance to the nova, the electron temperature, and the expansion velocity of the radio photosphere. As the ejecta expand, the density drops and the radio photosphere begins to recede, becoming optically thin at higher frequencies first. The flux density eventually peaks and starts to turn over at a particular frequency when the photosphere starts to recede. The time when the peak and turn over happen depends on the ejected mass, density profile, and electron temperature (see Figure 1; Hjellming et al. 1979; Seaquist \& Bode 2008). When the ejecta are completely optically thin, at a given frequency, the spectral index is flatter and ultimately $\alpha=-0.1$.

Over the last two decades, observations of novae in the radio have shown that our earlier assumption of a spherically symmetric expanding shell may not always be the case (see, e.g., Seaquist \& Palimaka 1977; Hjellming 1996; Seaquist \& Bode 2008; Roy et al. 2012 and references therein for extensive reviews on the subject). Seaquist \& Palimaka (1977) noted that solely looking at the radio light curve does not allow us to distinguish between a spherical shell and a polar shell (which are just portions of a spherical shell), in particular, to determine cone angles and the orientation of the polar shell. However, Heywood \& O'Brien (2007) applied spherically symmetric and ellipsoidal models to the eruption of V723 Cas, which was imaged with MERLIN, and could not find differences between these two models with a simulated $12 \mathrm{~h}$ track while on a $24 \mathrm{~h}$ track the ellipsoidal shell model could be retrieved during the optically thick phase. As the shell becomes optically thin, the images detect only the brighter emission coming from the innershell boundary. Historically, there have been no clear signatures of asymmetries from the radio light curves. Therefore, to break this degeneracy when determining the geometry from the radio light curve, complementary imaging is required (O'Brien et al. 2006; Sokoloski et al. 2008).

In the optical, nova ejecta have been resolved to show a myriad of structures far from spherical; these include bipolar morphologies and prolate structures with equatorial and tropical rings (e.g., Hutchings 1972; Solf 1983; Slavin et al. 1995; Gill \& O'Brien 1998, 2000; Bode 2002; Krautter et al. 2002; Harman \& O'Brien 2003; Ribeiro et al. 2009; Woudt et al. 2009). Furthermore, nebular line profiles are well replicated with bipolar geometries (e.g., Hutchings 1972; Gill \& O'Brien 1999; Ribeiro et al. 2013; Shore et al. 2013a, 2013b).

In this paper, we demonstrate the effects of bipolar shells on the radio light curves during a nova eruption. As commonly assumed in novae, expansion occurs into a vacuum and no intervening interstellar material is present, such as that expected from systems with strong winds (e.g., O'Brien et al. 2006; Chomiuk et al. 2012). Furthermore, we do not account for any other complicated morphologies, for example, as observed in V2672 Oph where there was a combination of a prolate structure-where the density appeared to decline faster-and polar and equatorial rings (Munari et al. 2011a). In keeping with previous literature, at radio frequencies, we have kept the assumption that the filling factor is unity and there is no clumpiness - we leave this as a discussion point later-we also assume instantaneous ejection. In Section 2, we describe our modeling procedures, starting from a spherically symmetric shell and then changing this to a bipolar shell. In Section 3, we present the results of this change and show the effect of varying different parameters on the radio light curve. Finally, in Section 4 , we discuss the relevance of our results and provide conclusions and look toward future work.

\section{MODELING PROCEDURE}

We aim to investigate the effect of the bipolar and nonhomogeneous structure of the ejecta on their spatially unresolved radio emission. To this aim, we construct an interactive, complex geometry of a nova ejecta in a three-dimensional interface within shape ${ }^{10}$ (Version 5; Steffen et al. 2011). The structure is then transferred to a three-dimensional grid on which radiation transfer is computed via ray tracing to the observer. Radiation transfer is based on emission and absorption coefficients which are provided as a function of physical parameters such as density, temperature, and wavelength. As the rays emerge from the grid, images and spectra are generated. Temporal evolution is simulated when a model of the structure's expansion is provided. The time sequence of the output is then generated automatically. The emissivity, used to generate the synthetic images, is proportional to the density squared.

In the Physics module within SHAPE, we input the free-free emission $\left(\epsilon_{v}\right.$ in units of $\left.\mathrm{W} \mathrm{m}^{-3} \mathrm{sr}^{-1} \mathrm{~Hz}^{-1}\right)$ and absorption $\left(\kappa_{v}\right.$ in units of $\mathrm{m}^{-1}$ ) coefficients at a given frequency ( $v$ in $\left.\mathrm{Hz}\right)$, as (Burke \& Graham-Smith 2009)

$$
\begin{gathered}
\epsilon_{v}=6.8 \times 10^{-51} Z^{2} T_{\mathrm{e}}^{-0.5} N_{\mathrm{e}} N_{\mathrm{z}} \bar{g}_{\mathrm{ff}}\left(v, T_{\mathrm{e}}\right) \exp -\frac{h v}{k T_{\mathrm{e}}}, \\
\kappa_{v}\left(T_{\mathrm{e}}\right)=1.77 \times 10^{-12} T_{\mathrm{e}}^{-1.5} Z^{2} N_{\mathrm{e}} N_{\mathrm{z}} v^{-2} \bar{g}_{\mathrm{ff}}\left(v, T_{\mathrm{e}}\right),
\end{gathered}
$$

respectively, where $N_{\mathrm{e}}=N_{z}$ are the electron and ion mass densities, $Z$ is the atomic number $(Z=1$ for a singly ionized atom), and $T_{\mathrm{e}}$ is the electron temperature. All values are in SI units. The Gaunt factor, $\bar{g}_{\mathrm{ff}}\left(v, T_{\mathrm{e}}\right)$, in the Rayleigh-Jeans approximation, $h v \ll k T_{\mathrm{e}}$, has only a logarithmic dependence on frequency (Bekefi 1966):

$$
\begin{gathered}
\bar{g}_{f f}\left(\nu, T_{\mathrm{e}}\right)=\frac{\sqrt{3}}{\pi}\left[17.7+\ln \frac{T_{e}^{1.5}}{v}\right] \\
\text { 2.1. Spherical Models }
\end{gathered}
$$

We first demonstrate that we can reproduce the classical spherical models within SHAPE. Our spherical model has a shell thickness of 0.25 , defined as the ratio of the inner radius to the outer radius of the shell. We define the inner radius to be $0.25 \times t \times V_{\max }$, where $t$ is the time since eruption and $V_{\max }$ is the maximum velocity; conversely, the outer radius is $t \times V_{\max }$. This assumes a velocity linearly proportional to the radius. The input parameters are $V_{\max }=3000 \mathrm{~km} \mathrm{~s}^{-1}$, the electron temperature, $T_{\mathrm{e}}=17,000 \mathrm{~K}$, ejected mass, $M_{\mathrm{ej}}=1 \times 10^{-4} M_{\odot}$, a $1 / r^{2}$ density distribution, and a distance of $1 \mathrm{kpc}$. These values were chosen primarily from radio observations (e.g., Hjellming et al. 1979; Hjellming 1996; Taylor et al. 1988; Heywood et al. 2005).

Monte Carlo line profile modeling of the structure of the nova ejecta assumes a $1 / r^{3}$ density profile (Shore et al. 2013a, 2013b), which is also used in photoionization models (e.g., Schwarz et al. 2001; Shore et al. 2003; Vanlandingham et al. 2005;

\footnotetext{
10 Available from http://www.astrosen.unam.mx/shape
} 

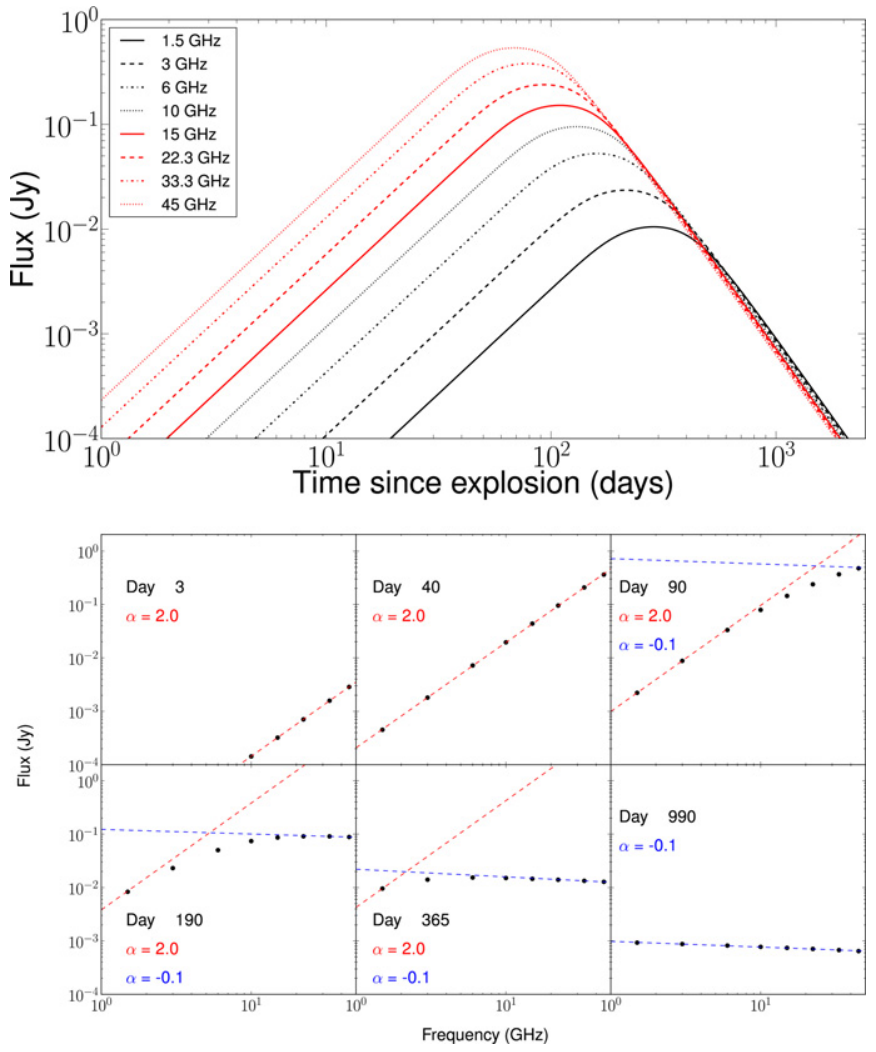

Figure 1. Top: synthetic radio light curves for a spherical eruption at a distance of $1 \mathrm{kpc}, M_{\mathrm{ej}}=1 \times 10^{-4} M_{\odot}, V_{\max }=3000 \mathrm{~km} \mathrm{~s}^{-1}, T_{\mathrm{e}}=17,000 \mathrm{~K}$, and the ratio of the inner to outer radius of the shell of 0.25 . The different frequency colors and line styles are as in the labels and Table 1. Bottom: spherical-model spectral evolution at different dates demonstrating how the spectral index changes from completely optically thick $(\alpha=2.0)$ to thin $(\alpha=-0.1)$. Furthermore, to guide the eye, lines are drawn to show the evolution of the spectral index from completely optically thick to thin.

(A color version of this figure is available in the online journal.)

Munari et al. 2011b). Meanwhile, for example, Munari et al. (2008) could not find a good fit using exponent values of 0 , -1 , and -3 with $1 / r^{2}$ providing a better fit, and morphokinematical modeling of the [O III] 4959/5007 $\AA$ emission line by Ribeiro et al. (2013) assumed a constant density distribution. The photoionization models above are based on CLOUDY (Ferland et al. 1998) which are in one dimension. The full three-dimensional treatment can be achieved, for example, with MOCCASIN (Ercolano et al. 2003), however, these are computationally intensive in order to explore the full parameter space. Pseudo-three-dimensional models based on CLOUDY are also being developed (RAINY3D; Moraes \& Diaz 2009, 2011) which can also account for clumpyness. We also note that a shell thickness of 0.25 is higher than that derived from photoionization modeling (e.g., Vanlandingham et al. 2005; Munari et al. 2008, 2011b)_although photoionization modeling should also be constrained with multifrequency and multi-epoch observations (e.g., Schwarz et al. 2001; Schwarz 2014)—and indeed also from recent geometrical studies of the resolved ejecta of GK Per (Liimets et al. 2012, although this object may be somewhat of a special case).

The frequencies explored are targeted toward observational bands of the Karl G. Jansky Very Large Array and are given in Table 1 while the results are presented in Figure 1. We compared the spherical models produced here with the numerical integration of a spherical shell modeled after Hjellming et al.
Table 1

Bandwidths Applied to the Models Based on those for the Karl, G. Jansky Very Large Array

\begin{tabular}{lccl}
\hline \hline Band & $\begin{array}{c}\text { Range } \\
(\mathrm{GHz})\end{array}$ & $\begin{array}{l}\text { Centre } \\
(\mathrm{GHz})\end{array}$ & Color/Linestlye \\
\hline $20 \mathrm{~cm}(L)$ & $1.0-2.0$ & 1.5 & Black/solid \\
$13 \mathrm{~cm}(S)$ & $2.0-4.0$ & 3.0 & Black/dashed \\
$6 \mathrm{~cm}(C)$ & $4.0-8.0$ & 6.0 & Black/dashdot \\
$3 \mathrm{~cm}(X)$ & $8.0-12.0$ & 10.0 & Black/dotted \\
$2 \mathrm{~cm}(\mathrm{Ku})$ & $12.0-18.0$ & 15.0 & Red/solid \\
$1.3 \mathrm{~cm}(K)$ & $18.0-26.5$ & 22.3 & Red/dashed \\
$1 \mathrm{~cm}(\mathrm{Ka})$ & $26.5-40.0$ & 33.3 & Red/dashdot \\
$0.7 \mathrm{~cm}(Q)$ & $40.0-50.0$ & 45.0 & Red/dotted \\
\hline
\end{tabular}

Table 2

Best-fit Spherical Model in the $S$ and $Q$ bands for Different Values of the squeeze, Assuming Input Models at a Distance of $1 \mathrm{kpc}, M_{\mathrm{ej}}=1 \times 10^{-4} M_{\odot}$, $T_{\mathrm{e}}=17,000 \mathrm{~K}, V_{\max }=3000 \mathrm{~km} \mathrm{~s}^{-1}$, and a Shell Thickness of 0.25

\begin{tabular}{|c|c|c|c|c|c|}
\hline Squeeze & $\begin{array}{c}i \\
(\mathrm{deg})\end{array}$ & $\begin{array}{c}T_{\mathrm{e}} \\
\left(\times 10^{4} \mathrm{~K}\right)\end{array}$ & $\begin{array}{c}M_{\mathrm{ej}} \\
\left(\times 10^{-4} M_{\odot}\right)\end{array}$ & Shell & $\begin{array}{l}\text { Reduced } \chi^{2} \\
(\text { dof }=399)\end{array}$ \\
\hline 0.0 & - & 1.73 & 0.98 & 0.24 & 0.47 \\
\hline \multirow[t]{2}{*}{0.1} & 0 & 1.42 & 1.04 & 0.25 & 0.68 \\
\hline & 90 & 1.64 & 1.03 & 0.23 & 0.40 \\
\hline \multirow[t]{2}{*}{0.2} & 0 & 1.17 & 1.11 & 0.26 & 1.73 \\
\hline & 90 & 1.54 & 1.09 & 0.22 & 0.42 \\
\hline \multirow[t]{2}{*}{0.3} & 0 & 0.99 & 1.18 & 0.25 & 3.19 \\
\hline & 90 & 1.43 & 1.16 & 0.20 & 0.56 \\
\hline \multirow[t]{2}{*}{0.4} & 0 & 0.84 & 1.27 & 0.24 & 4.54 \\
\hline & 90 & 1.33 & 1.23 & 0.18 & 0.95 \\
\hline \multirow[t]{2}{*}{0.5} & 0 & 0.72 & 1.37 & 0.21 & 6.07 \\
\hline & 90 & 1.23 & 1.32 & 0.16 & 1.73 \\
\hline \multirow[t]{2}{*}{0.6} & 0 & 0.63 & 1.47 & 0.17 & 8.27 \\
\hline & 90 & 1.12 & 1.42 & 0.13 & 3.52 \\
\hline \multirow[t]{2}{*}{0.7} & 0 & 0.55 & 1.62 & 0.13 & 14.46 \\
\hline & 90 & 1.01 & 1.57 & 0.10 & 7.14 \\
\hline \multirow[t]{2}{*}{0.8} & 0 & 0.48 & 1.81 & 0.08 & 36.24 \\
\hline & 90 & 0.90 & 1.77 & 0.06 & 22.46 \\
\hline \multirow[t]{2}{*}{0.9} & 0 & 0.42 & 2.18 & 0.04 & 128.37 \\
\hline & 90 & 0.78 & 2.17 & 0.03 & 58.01 \\
\hline
\end{tabular}

Note. When fitting the models, we kept the distance and $V_{\max }$ constant.

(1979) and Heywood et al. (2005). To demonstrate that the models developed in this paper are equivalent to those previously published in the first row in Table 2, we show the fit of one such model to a spherical model from this paper.

\subsection{Bipolar Models}

Subsequently, we modify our spherical shell to a bipolar geometry, where the ratio of the major axis is five times greater than the minor axis (left-hand panel, Figure 2). All other system parameters are kept the same as in the spherical case. In this bipolar case, the maximum velocity along the major axis is $V_{\max }=3000 \mathrm{~km} \mathrm{~s}^{-1}$, while in the minor axis it is $V_{\text {minor }}=$ $600 \mathrm{~km} \mathrm{~s}^{-1}$, determined from the ratio of the axes. We use the squeeze modifier to obtain the different axial ratios and is defined as squeeze $=1-a / b$, where $a$ and $b$ are the semi-minor and -major axes, respectively. 

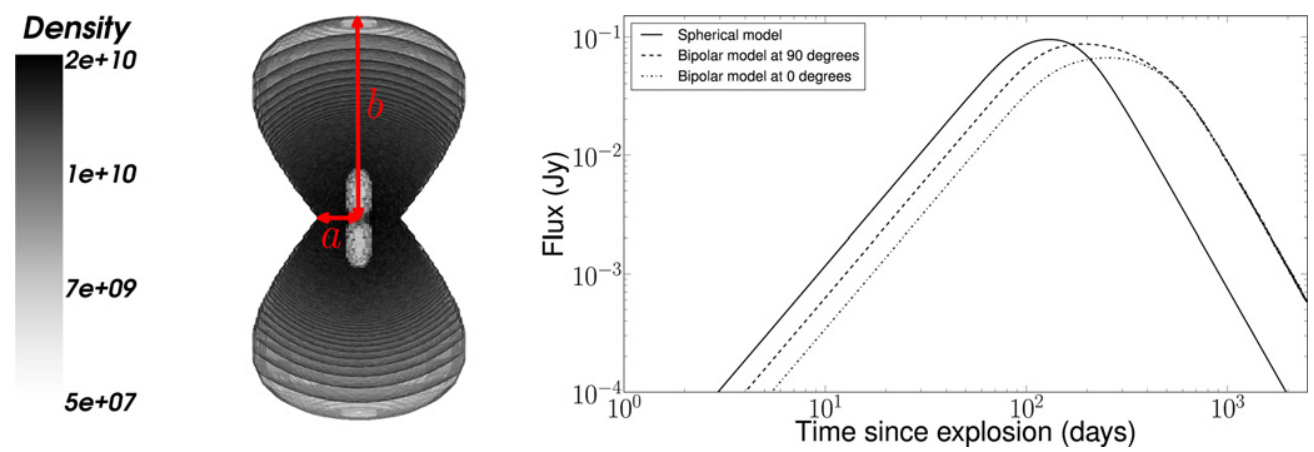

Figure 2. Left: bipolar morphology used as input for the modeling, as seen at $90^{\circ}$. The density is in electrons per cubic meter. The ratio of the semi-major to semi-minor axis ( $a$ and $b$, respectively) is of 5 (squeeze $=0.8$ ) at $90^{\circ}$ as in the text. Right: comparison between the $X$-band spherical model (solid black; as Figure 1) and a bipolar model, with the same initial parameters as the spherical model at $0^{\circ}$ and $90^{\circ}$ (black dash-dotted and dashed lines, respectively).

(A color version of this figure is available in the online journal.)
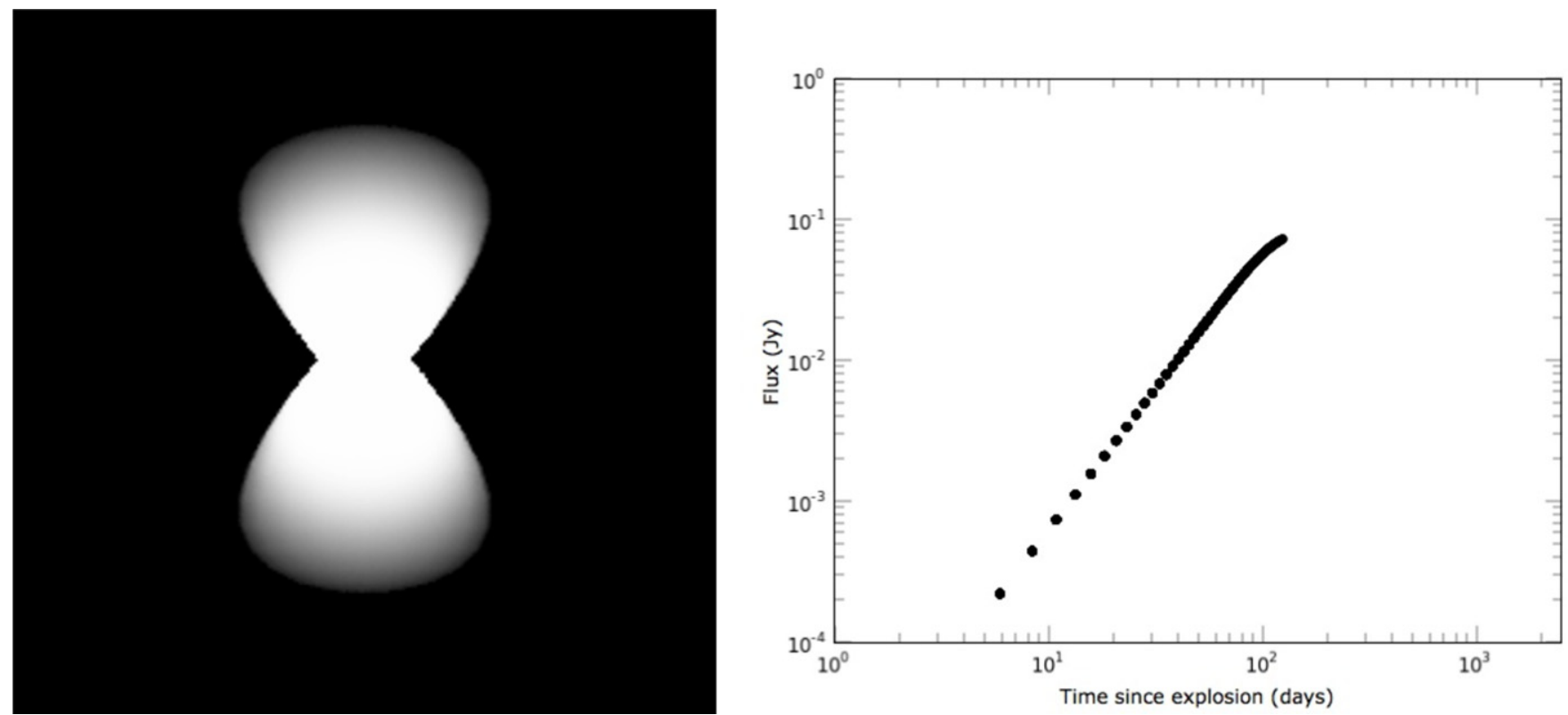

Figure 3. Synthetic image and light curve (left and right panels, respectively) for the evolution of the bipolar nova shell, given in Figure 2, as it expands from the optically thick phase to the optically thin decline, at an inclination of $90^{\circ}$.

(An animation of this figure is available in the online journal.)

\section{RESULTS}

The right-hand panel of Figure 2 shows the synthetic light curve for the bipolar model at two different inclinations (where an inclination $i=90^{\circ}$ corresponds to the orbital plane being edge-on, and $i=0^{\circ}$ being face-on) compared with the spherical model in the $X$ band $(10 \mathrm{GHz})$ and assuming the initial conditions as described in the previous section. Below, we describe the evolution of the bipolar-model synthetic light curve, and in Figure 3 we provide a time sequence of the evolution for a bipolar system at an inclination angle of $90^{\circ}$ as a visual aid to the description.

1. The initial optically thick rise phases are equivalent for both the spherical and bipolar models and follow the Planck function. However, the flux density is lower in the bipolar models due to the fact that it is proportional to the surface area of the shell (as viewed on the plane of the sky); in the bipolar model, depending on the inclination angle, only a certain fraction of the object is observed as compared to a spherical ejecta at the same phase of evolution. Furthermore, the flux density at this stage increases as $t^{2}$ in all three cases-spherical and bipolar. Again, the spec- tral index at this time is $\alpha=2.0$ (see the upper right panel of Figure 4 and the lower panel of Figure 1 for comparison).

2. For the same mass of ejecta, the bipolar-model density will obviously be higher due to the fact that the volume is smaller. Depending on the inclination angle for the bipolar model, the peak flux density is around the same level or slightly below ( $90^{\circ}$ and $0^{\circ}$, respectively). The lower flux density, at $0^{\circ}$, is due to the fact that the photospheres never reach as large an area as if the ejecta where observed, for example, at $90^{\circ}$.

3. The light curve then enters a "plateau" phase while the photosphere recedes, which is dependent on the shell thickness and the squeeze-both reducing the length of the plateau for a decrease in the shell thickness and the degree of bipolarity (bottom panels in Figure 4) - before entering the internal cavity in the ejecta and declining. The decline phase flux density is proportional to $t^{-3}$ and follows the same behavior as the spherical spectral index, $\alpha=$ -0.1 (upper right panel, Figure 4), albeit at slightly higher flux density.

Furthermore, in the Appendix, we show the effects that changing various parameters have on the radio light curves. 

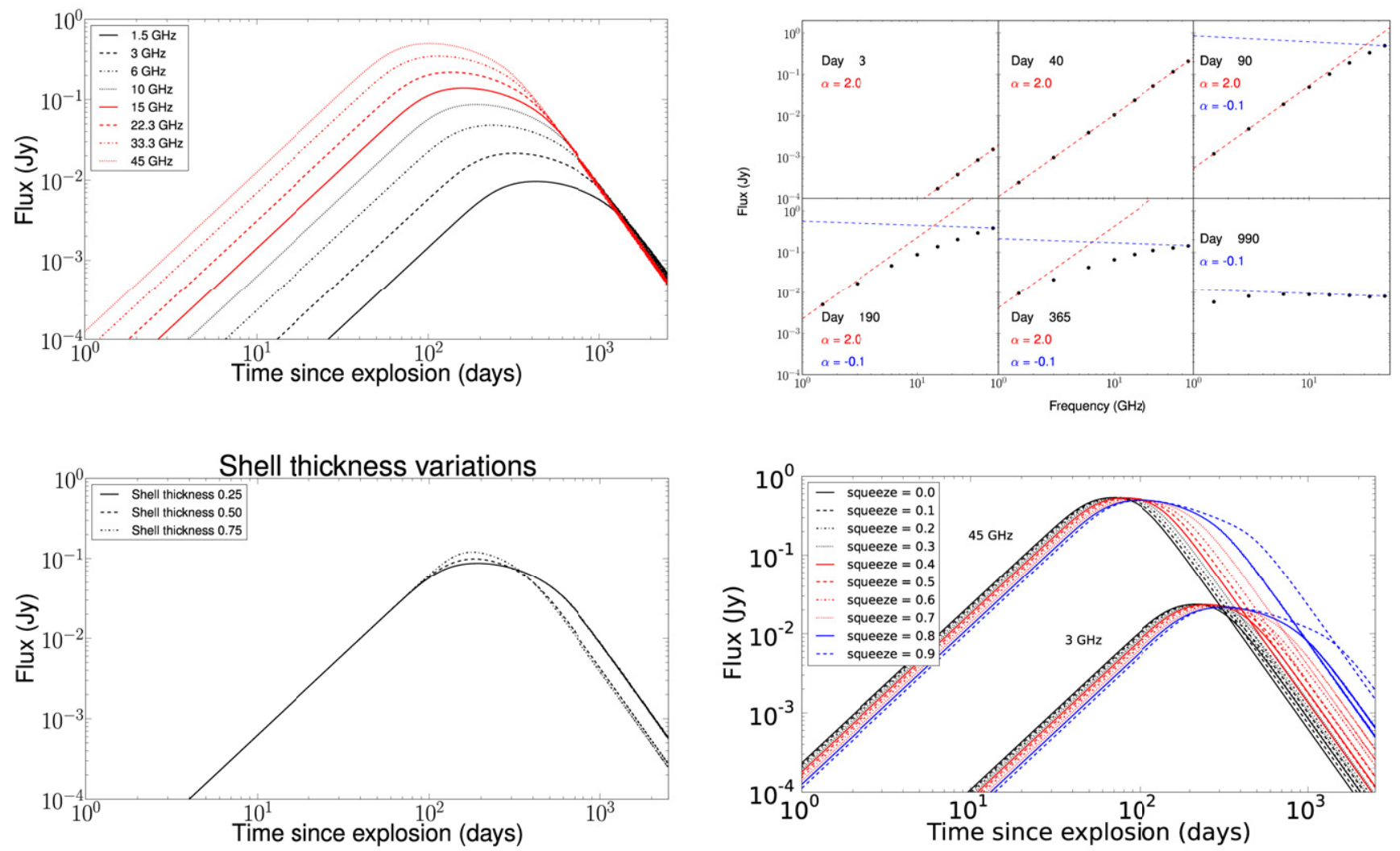

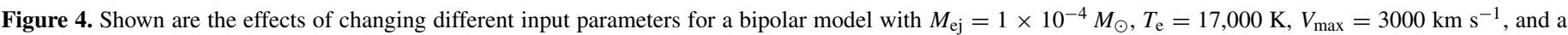

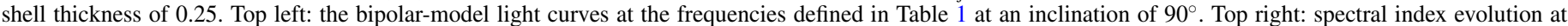

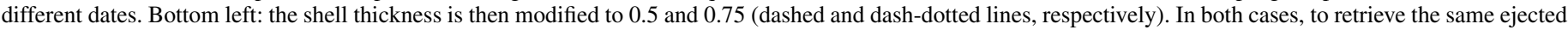

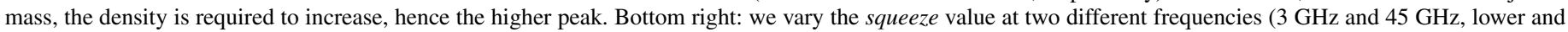
upper curves, respectively). It is notable that moving from a sphere (squeeze $=0.0$ ) to a bipolar morphology increases the "plateau" phase.

(A color version of this figure is available in the online journal.)

In all cases, we start with the $X$-band model and then change one parameter at a time. As in the spherical case, the higher the frequency the higher the flux densities and the light curve peaks earlier. Decreasing the ejected mass results in lower peak densities and earlier turnover as expected. Similarly, the higher the temperature the earlier the peak. Increasing the velocity causes the material to become optically thin earlier with higher flux density.

Finally, we fit spherical-model synthetic radio light curves to the bipolar-model synthetic radio light curves at $0^{\circ}$ and $90^{\circ}$. We apply the same initial conditions as before, $M_{\mathrm{ej}}=1 \times 10^{-4} M_{\odot}$, $T_{\mathrm{e}}=17,000 \mathrm{~K}, V_{\max }=3000 \mathrm{~km} \mathrm{~s}^{-1}$, and a shell thickness of 0.25 , at a distance of $1 \mathrm{kpc}$. The results are shown in Table 2, and in Figure 5 we show fits to the synthetic light curves for a squeeze of 0.9. The general upshot of these results is that if we fit a spherical model to a bipolar-model light curve, then we find an artificially high ejected mass, reduced temperature, and increased width of the shell, keeping the maximum velocity and distance the same. As illustrated in Table 2, the larger the departure from sphericity and the lower the inclination, the greater the difference. Furthermore, we show in Table 2 the results of the fit to a sphere (squeeze $=0.0$ ) to demonstrate the stability of the fitting.

\section{DISCUSSION AND CONCLUSIONS}

First and foremost, the results presented above show some remarkable similarities in the light curve between the different model morphologies. One notable difference, however, is the

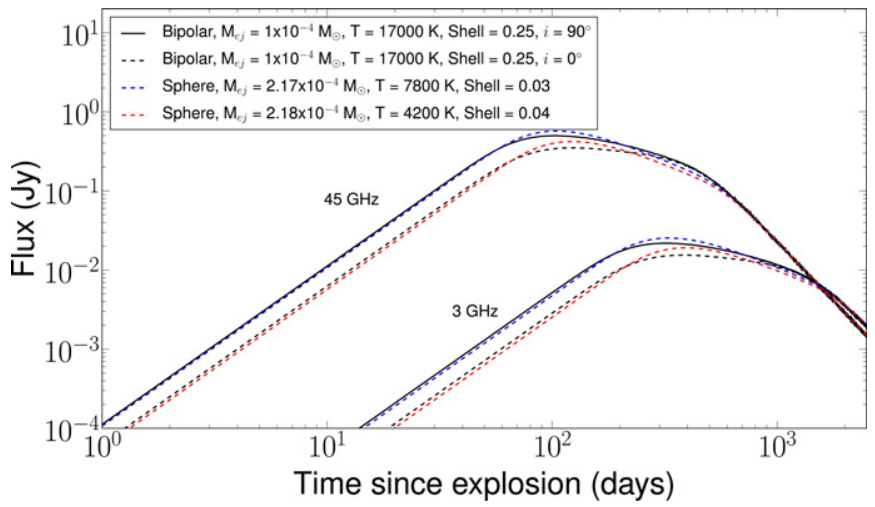

Figure 5. Best-fit spherical-model synthetic light curves are fitted to bipolarmodel synthetic light curves. The input bipolar model at $0^{\circ}$ and $90^{\circ}$ (dashed and solid black lines, respectively) assumes $M_{\mathrm{ej}}=1 \times 10^{-4} M_{\odot}, T_{\mathrm{e}}=17,000 \mathrm{~K}$, $V_{\max }=3000 \mathrm{~km} \mathrm{~s}^{-1}$, and a relative shell thickness of 0.25 at a distance of $1 \mathrm{kpc}$. The best-fit spherical models are shown as blue and red dashed lines for the bipolar models at $90^{\circ}$ and $0^{\circ}$, respectively. The result shows that to find the best fit while keeping the maximum expansion velocity and distance constant, we require that the ejected mass double increase the shell size (so that it reaches closer to the explosion site), and, depending on the inclination, reduce the temperature by more than half.

(A color version of this figure is available in the online journal.)

shape of the light curve itself-depending on the details of the shell thickness and the ratio of the minor- to major-axis. Disentangling the geometry and system parameters from the radio light curve is difficult without further information from 
different wavelengths. If the light curve presents a longer "plateau" phase, as observed in Figures 2 and 4, then we may assume that this is an indication of a bipolar morphology. As shown in the lower left panel of Figure 4, we are able to reduce the "plateau" phase if we reduce the size of the shell. Therefore, all of these factors will induce an error in the mass estimation, and hence it is imperative that we apply estimates of the ejecta geometry derived from optical line profiles or high-resolution imaging to the radio observations.

The results presented in Table 2 and Figure 5 demonstrate that in some cases in the literature, we may have overestimated the ejected masses by fitting spherical models to light curves that arise from a bipolar ejecta and underestimated the temperature of the ejecta. We require, therefore, that the geometry of the system is constrained soon after eruption (see, e.g., Ribeiro et al. 2013; Shore et al. 2013b). These results are a stepping stone toward solving the question of the order-of-magnitude discrepancy between the predicted and observed ejected masses (the observed being the higher masses; Prialnik \& Kovetz 1995; Gehrz et al. 1998; Starrfield et al. 1998; José et al. 1999; Gehrz 2002; Yaron et al. 2005). There are a number of issues that will affect the ejected masses; i.e., clumpiness, the filling factor, and a realistic ejecta morphology (as described above the morphology is far from simplistic as a simple bipolar too) among others factors. The discrepancy appears to be predominately in the fastest novae (see, e.g., Roy et al. 2012).

These simple models of a bipolar morphology assuming a free-free emission process, however, are not sufficient to replicate the myriad of observed radio light curves. For example, V1723 Aql presents a steep rise $\left(S_{v} \propto t^{3.3}\right)$ during the optically thick phase (Krauss et al. 2011; Weston et al. 2013). Furthermore, the temporal and spectral evolution is different from that described in this paper. The radio light curves also show bumps (e.g., V1324 Sco; Weston et al. 2013) where there is a phase at which the flux density increases then falls, only subsequently to rise again before its final decline; kinematically, we may understand this to arise from two distinct shells where the fastest-moving shell becomes optically thin first and as the radio photosphere recedes toward the inner shell, that is still optically thick, the flux density rises again once the first shell becomes completely optically thin (we leave this for a future work) - this may also explain some of the features observed in T Pyx, for example, as observed in Nelson et al. (2014). However, the Russian doll structure described above does not account for the steep rise in the radio light curve, which may be due to a number of factors (for example, a variable temperature gradient in the ejecta left for future work). Indeed, current theoretical nova models do not predict a series of discrete, timeseparated mass ejections, however, Shore (2013) has presented a model for the spectral and photometric evolution that does not require secondary ejection or winds. In terms of future observations, we require early, frequent temporal and spectral evolution of these sources with good enough time sampling. This may be achieved with upcoming large radio surveys, such as ThunderKAT on MeerKAT (a precursor telescope to the Square Kilometer Array). Furthermore, with improvements to very long baseline interferometry, we are able to resolve sources much earlier and with smaller angular scales than before, which will provide clues to the origin of they myriad of radio light curves.

In this paper, we aim to show the effects bipolar models have on our understanding of radio nova light curves. We show the effects that changing various parameters have on the radio light curve, and our main conclusion is that in some cases where spherical models have been fit to an eruption where bipolar geometries in fact are present, this may induce an error in overestimating the mass of a factor of two. An immediate example is that of V703 Cas. Heywood et al. (2005) interpreted the light curve as arising from a spherical model and retrieved parameters, namely, the mass and distance to the nova. The spherical model was later shown to be incorrect when Lyke \& Campbell (2009) concluded that the morphology of the ejecta was different in the different emission lines and suggested different ejection events. Lyke \& Campbell (2009) also derived a revised distance to V723 Cas from the expansion parallax method, suggesting the object was much closer than that derived from Heywood et al. (2005). We are now building models to account for these changes to update the parameters of V723 Cas (V. A. R. M. Ribeiro et al., in preparation).

Finally, in this first paper, we kept the radio models as simple and similar to those already present in the literature, at least at radio frequencies. A number of effects that were not consider but warrant some discussion include non-uniformity of the ejecta, both in terms of the filling factor and clumpyness as well as temperature variations in the ejecta. The ejecta, particularly at optical wavelengths, have been shown to be very clumpy (e.g., HR Del, GK Per, RR Pic, T Pyx, AT Cnc; Gill \& O'Brien 1998; Harman \& O'Brien 2003; Liimets et al. 2012; Shara et al. 1997, 2012a, 2012b; Slavin et al. 1995). Williams (1994) had already suggested that the ejecta shell is not homogeneous, as measured from the optical line ratios of $\left[\mathrm{O}_{\mathrm{I}}\right]$ and that neutral gas could exist in clumps. The clumps may be formed from Rayleigh-Taylor instabilities (Lloyd et al. 1997) during the early phases, while at later stages, when the shell density decreases, Kelvin-Helmholtz instabilities should occur (Chevalier et al. 1992; Casanova et al. 2011). Moraes \& Diaz (2009, 2011) showed that the presence of clumps in non-spherical shells can affect the mass determination by a factor of $\sim$ five. Finally, until now the temperature has been assumed to be constant throughout the shell, however, there is strong evidence that this is not always the case. Metzger et al. (2014) have modeled V1324 Sco from a one-dimensional model in terms of shocks between a fast nova outflow and a dense external shell setting up a temperature gradient. In the Metzger et al. (2014) model, they account for shocks and the ionization state of the medium, replicating with confidence the radio light curve.

V.A.R.M.R. thanks Tom Jarrett for providing computing facilities through the NRF SARChI, Brian Warner for reading an initial draft and for useful discussions, and Russ Taylor for useful discussions. V.A.R.M.R. acknowledges the South African SKA Project for funding the postdoctoral fellowship position at the University of Cape Town (UCT). T.S. acknowledges the National Society of Black Physicists, USA, for funding the PhD studentship at UCT. P.A.W. kindly acknowledges support from UCT and the NRF. We are grateful to our referee, Steven Shore, for very constructive comments. The National Radio Astronomy Observatory is a facility of the National Science Foundation operated under coorperative agreement by Associated Universities, Inc.

\section{APPENDIX}

\section{VARIATIONS IN MASS, TEMPERATURE, AND VELOCITY FOR A BIPOLAR MODEL}

Figure 6 shows the effects of changing a number of input parameters. Changing the ejected mass to higher values will 

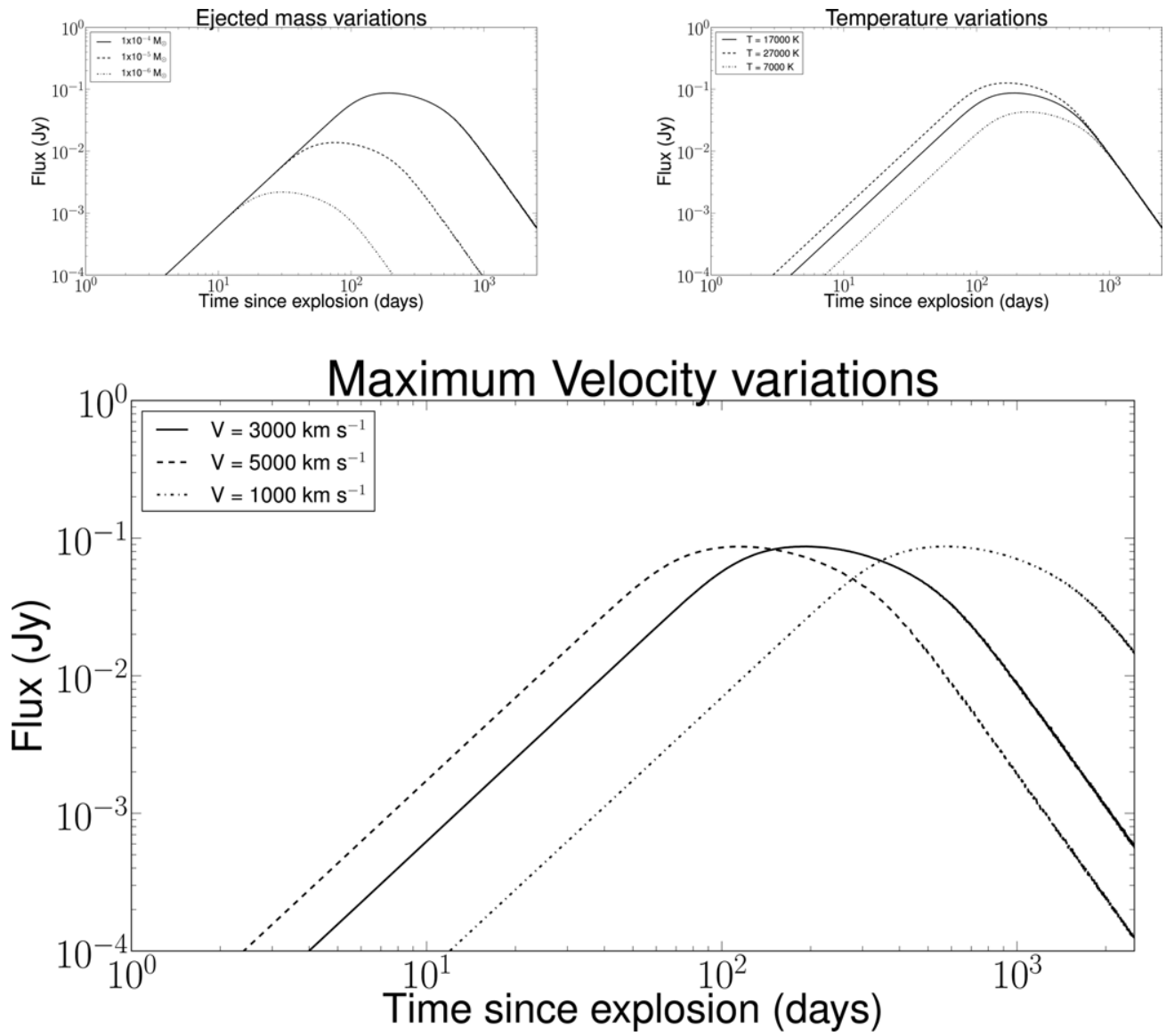

Figure 6. Effects of changing different input parameters are shown for a bipolar model with $M_{\mathrm{ej}}=1 \times 10^{-4} M_{\odot}, T_{\mathrm{e}}=17,000 \mathrm{~K}, V_{\max }=3000 \mathrm{~km} \mathrm{~s}{ }^{-1}$, and a relative shell thickness of 0.25 (solid lines). Top left: changing the ejected mass to $1 \times 10^{-5} M_{\odot}$ and $1 \times 10^{-6} M_{\odot}$ (dashed and dash-dotted lines, respectively) show a decrease in the peak flux density as well as an earlier turn over. Top right: the temperature was varied to 27,000 and $7000 \mathrm{~K}$ (dashed and dash-dotted lines, respectively). Bottom: modifying the velocity to 5000 and $1000 \mathrm{~km} \mathrm{~s}^{-1}$ (dashed and dash-dotted lines, respectively) shifts the peak density to earlier or later times, respectively.

increase the flux density and cause a later peak/turnover as the material in the ejecta stays optically thick for longer. Increasing the temperature will shift the radio light curve to higher flux densities and a higher, and earlier, peak/turnover. While increasing the velocity will cause the radio light curve to shift to an earlier peak/turnover, but at exactly the same peak flux density. These effects have exactly the same behavior in a spherical model.

\section{REFERENCES}

Bekefi, G. 1966, in Radiation Processes in Plasmas, (New York: Wiley),

Bode, M. F. 2002, in AIP Conf. Proc. 637, Classical Nova Explosions, ed. M. Hernanz \& J. José (Melville, Ny: AIP), 497

Bode, M. F. 2010, AN, 331, 160

Bode, M. F., \& Evans, A. (ed.) 2008, Classical Novae (2nd ed; Cambridge: Cambridge Univ. Press)

Burke, B. F., \& Graham-Smith, F. 2009, An Introduction to Radio Astronomy (Cambrigde: Cambrigde Univ. Press)

Casanova, J., José, J., García-Berro, E., Shore, S. N., \& Calder, A. C. 2011, Natur, 478, 490

Chevalier, R. A., Blondin, J. M., \& Emmering, R. T. 1992, ApJ, 392, 118

Chomiuk, L., Krauss, M. I., Rupen, M. P., et al. 2012, ApJ, 761, 173

Di Stefano, R., Orio, M., \& Moe, M. 2013, in IAU Symp. 281, Binary Paths to Type Ia Supernovae Explosions (IAU S281; Cambridge: Cambridge Univ. Press)

Ercolano, B., Barlow, M. J., Storey, P. J., \& Liu, X.-W. 2003, MNRAS, 340,1136

Ferland, G. J., Korista, K. T., Verner, D. A., et al. 1998, PASP, 110, 761

Gehrz, R. D. 2002, in AIP Conf. Proc. 637, Classical Nova Explosions, ed. M. Hernanz \& J. José (Melville, NY: AIP), 198
Gehrz, R. D., Truran, J. W., Williams, R. E., \& Starrfield, S. 1998, PASP, 110, 3 Gill, C. D., \& O'Brien, T. J. 1998, MNRAS, 300, 221

Gill, C. D., \& O’Brien, T. J. 1999, MNRAS, 307, 677

Gill, C. D., \& O’Brien, T. J. 2000, MNRAS, 314, 175

Harman, D. J., \& O'Brien, T. J. 2003, MNRAS, 344, 1219

Heywood, I., \& O'Brien, T. J. 2007, MNRAS, 379, 1453

Heywood, I., O’Brien, T. J., Eyres, S. P. S., Bode, M. F., \& Davis, R. J. 2005, MNRAS, 362, 469

Hjellming, R. M. 1996, in ASP Conf. Ser. 93, Radio Emission from the Stars and the Sun, ed. A. R. Taylor \& J. M. Paredes (San Francisco, CA: ASP), 174

Hjellming, R. M., \& Wade, C. M. 1970, ApJL, 162, L1

Hjellming, R. M., Wade, C. M., Vandenberg, N. R., \& Newell, R. T. 1979, AJ, 84,1619

Hutchings, J. B. 1972, MNRAS, 158, 177

José, J., Coc, A., \& Hernanz, M. 1999, ApJ, 520, 347

Krauss, M. I., Chomiuk, L., Rupen, M., et al. 2011, ApJL, 739, L6

Krautter, J., Woodward, C. E., Schuster, M. T., et al. 2002, AJ, 124, 2888

Kwok, S. 1983, MNRAS, 202, 1149

Liimets, T., Corradi, R. L. M., Santander-García, M., et al. 2012, ApJ, 761, 34

Lloyd, H. M., O'Brien, T. J., \& Bode, M. F. 1997, MNRAS, 284, 137

Lyke, J. E., \& Campbell, R. D. 2009, AJ, 138, 1090

Metzger, B. D., Hascoet, R., Vurm, I., et al. 2014, MNRAS, 442, 713

Moraes, M., \& Diaz, M. 2009, AJ, 138, 1541

Moraes, M., \& Diaz, M. 2011, PASP, 123, 844

Munari, U., Ribeiro, V. A. R. M., Bode, M. F., \& Saguner, T. 2011a, MNRAS, 410,525

Munari, U., Siviero, A., Dallaporta, S., et al. 2011b, NewA, 16, 209

Munari, U., Siviero, A., Henden, A., et al. 2008, A\&A, 492, 145

Nelson, T., Chomiuk, L., Roy, N., et al. 2014, ApJ, 785, 78

Newsham, G., Starrfield, S., \& Timmes, F. 2013, in ASP Conf. Ser., Stella Novae: Past and Future Decades, ed. P. A. Woudt \& V. A. R. M. Ribeiro (San Francisco, CA: ASP), in press (arXiv: 1303.3642)

O’Brien, T. J., Bode, M. F., Porcas, R. W., et al. 2006, Natur, 442, 279 
Prialnik, D., \& Kovetz, A. 1995, ApJ, 445, 789

Ribeiro, V. A. R. M., Bode, M. F., Darnley, M. J., et al. 2009, ApJ, 703, 1955

Ribeiro, V. A. R. M., Munari, U., \& Valisa, P. 2013, ApJ, 768, 49

Ritossa, C., Garcia-Berro, E., \& Iben, I., Jr. 1996, ApJ, 460, 489

Roy, N., Chomiuk, L., Sokoloski, J. L., et al. 2012, BASI, 40, 293

Schwarz, G. 2014, in ASP Conf. Ser., Stella Novae: Past and Future Decades, ed. P. A. Woudt \& V. A. R. M. Ribeiro (San Francisco, CA: ASP), in press

Schwarz, G. J., Shore, S. N., Starrfield, S., et al. 2001, MNRAS, 320, 103

Seaquist, E. R., \& Bode, M. F. 2008, in Classical Novae, ed. M. F. Bode \& A. Evans (2nd ed; Cambridge: Cambridge Univ. Press), 141

Seaquist, E. R., \& Palimaka, J. 1977, ApJ, 217, 781

Shara, M. M., Mizusawa, T., Wehinger, P., et al. 2012a, ApJ, 758, 121

Shara, M. M., Zurek, D., De Marco, O., et al. 2012b, AJ, 143, 143

Shara, M. M., Zurek, D. R., Williams, R. E., et al. 1997, AJ, 114, 258

Shore, S. N. 2013, A\&A, 559, L7

Shore, S. N., De Gennaro Aquino, I., Schwarz, G. J., et al. 2013a, A\&A, 553, A123

Shore, S. N., Schwarz, G., Bond, H. E., et al. 2003, AJ, 125, 1507

Shore, S. N., Schwarz, G. J., De Gennaro Aquino, I., et al. 2013b, A\&A, 549, A 140

Slavin, A. J., O’Brien, T. J., \& Dunlop, J. S. 1995, MNRAS, 276, 353
Sokoloski, J. L., Rupen, M. P., \& Mioduszewski, A. J. 2008, ApJL, 685, L137

Solf, J. 1983, ApJ, 273, 647

Starrfield, S., Iliadis, C., \& Hix, W. R. 2008, in Classical Novae, ed. M. F. Bode \& A. Evans (2nd ed; Cambridge: Cambridge Univ. Press), 77

Starrfield, S., Sparks, W. M., \& Truran, J. W. 1985, ApJ, 291, 136

Starrfield, S., Truran, J. W., Wiescher, M. C., \& Sparks, W. M. 1998, MNRAS, 296, 502

Steffen, W., Koning, N., Wenger, S., Morisset, C., \& Magnor, M. 2011, IEEE Trans. Visualization Comp. Graphics, 17, 454

Taylor, A. R., Hjellming, R. M., Seaquist, E. R., \& Gehrz, R. D. 1988, Natur, 335,235

Truran, J. W., \& Livio, M. 1986, ApJ, 308, 721

Vanlandingham, K. M., Schwarz, G. J., Shore, S. N., Starrfield, S., \& Wagner, R. M. 2005, ApJ, 624, 914

Wade, C. M., \& Hjellming, R. M. 1971, ApJL, 163, L65

Weston, J. H. S., Sokoloski, J. L., Zheng, Y., et al. 2013, in ASP Conf. Ser., Stella Novae: Past and Future Decades, ed. P. A. Woudt \& V. A. R. M. Ribeiro (San Francisco, CA: ASP), arXiv:1306.2265

Williams, R. E. 1994, ApJ, 426, 279

Woudt, P. A., Steeghs, D., Karovska, M., et al. 2009, ApJ, 706, 738

Yaron, O., Prialnik, D., Shara, M. M., \& Kovetz, A. 2005, ApJ, 623, 398 